\title{
PAR-1 helps Wnt to get rid of JNK
}

Wnt/Wingless (Wg) signalling is critical for normal embryonic development, and deregulation of the Wnt pathway can lead to aberrant development or cancer. The Dishevelled (Dsh) protein is phosphorylated and activated as a result of Wnt-binding to its receptor Frizzled $(\mathrm{Fz})$. This in turn is required for activation of the two possible effectors of Wnt signalling, $\beta$-catenin and c-Jun $\mathrm{N}$-terminal kinase (JNK). Dsh activation of JNK is also involved in the control of cell polarity. Sun et al. now report in this issue (p. 628) on differential regulation by Wnt of Dsh activation of $\beta$-catenin and JNK through the PAR-1 kinase. This represents a new function for PAR-1, which also controls cell polarity in asymmetric cell division in Caenorhabditis elegans and in Drosophila.

Sun et al. purified PAR-1 as a Dsh-associated kinase. They showed that PAR-1 phosphorylates Dsh in vitro and in vivo, and that Wnt signalling results in increased PAR-1 activity. Overexpression of PAR-1 has differential effects on the Wnt/ $\beta$ catenin and $\mathrm{Wnt} / \mathrm{JNK}$ pathways as it enhances $\mathrm{Wnt} / \beta$-catenin signalling while inhibiting Wnt/JNK signalling. Conversely, antisense par-1 oligonucleotides inhibit Wnt/ $\beta$-catenin signalling in mammalian cells, and RNA interference with double-stranded par-1 RNA generates a $w g$ loss-of-function-like phenotype. A kinase-dead mutant of PAR-1 and a peptide that corresponds to the Dsh-binding domain of PAR-1 dominantly inhibit endogenous PAR-1 activity and phosphorylation of Dsh and $\mathrm{Wnt} / \beta$-catenin signalling induced by Wnt, although they fail to

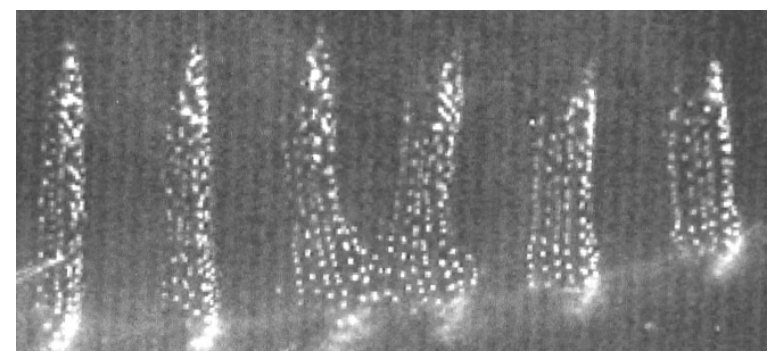

L. T. WILLIAMS AND COLLEAGUES

inhibit Wnt/JNK signalling. Hence, PAR-1 seems to act as a 'switch' between the Wnt/ $\beta$-catenin and Wnt/JNK pathways. The dominant-negative PAR-1 constructs does not interfere with the transcriptional response to overexpression of $\beta$-catenin, which indicates that PAR-1 indeed acts upstream of $\beta$-catenin.

These results indicate that Wnt signalling and cell and embryo polarity might have overlapping molecular mechanisms, and that Dsh might be one of the relevant targets of PAR-1 in regulating polarity as well as in Wnt signalling.

VALERIE DEPRAETERE this RIP1Tag2 system, Cavallaro et al. show that in addition to E-cadherin, loss of regulators of cell-matrix interaction might work in tumour progression, and that the two events do not seem to be directly linked. N-CAM, which mediates homoand heterotypic cell-cell adhesion, is considerably downregulated, and changes from the isoform N-CAM120 (relative molecular mass of 120,000) to the alternatively spliced N-CAM140 and N-CAM180 isoforms in advanced tumours in the RIP1Tag2 tumour model. Furthermore, restoration of $\mathrm{N}$-CAM120 in N-CAM-deficient mice prevents tumour metastasis. This indicates that only the extracellular domain is needed for assembly of the signalling complex.

Loss of N-CAM expression also correlates with increased malignancy and poor prognosis of certain gastrointestinal cancers, including pancreatic cancer ${ }^{12}$. FGFR-4 is predominantly expressed in endodermally derived tissues, such as in cells of the gastrointestinal tract during embryonic development, and is downregulated postnatally ${ }^{2}$. However, several carcinomas have elevated FGFR-4 expression, including pancreatic adenocarcinomas. Interestingly, FGFR-4 is not expressed in the early hyperplastic $\beta$-cell islets, but appears only in the more advanced tumours ${ }^{13}$. Even the loss of one N-CAM allele, which leads to considerable downregulation of expression, is asso- ciated with lymphatic metastasis; this is consistent with the idea that a critical threshold of expression is needed for signalling ${ }^{5,14}$. The presence of a multicomponent signalling complex indicates that downregulation of other components might produce similar effects.

Although RIP1Tag $2 \times$ FGFR4 $^{-/-}$mice have been generated and multiple parameters of malignancy reported, tumor metastasis was not reported in these mice ${ }^{13}$. The results of Cavallaro et al. also indicate that FGFR-4 signals may activate $\beta_{1}$-integrin as one specific target for increased cell-matrix adhesion at focal contacts. Although other adhesion mechanisms regulated by bovine FGFs are as yet poorly defined, FGF induced neovascularization depends on activation of $\alpha_{v} \beta_{3}$-integrin specifically in the angiogenic endothelial sprouts, and these events are also mediated through the c-Src tyrosine kinase, as in the present model ${ }^{15}$.

The loss of cell-matrix interaction in $\mathrm{N}$-CAM-deficient insulinomas leads to a growth pattern of the insulinoma cells in clusters, with haemorrohagic lacunae in between. This may create a 'washout' effect, whereby the interstitial pressure, which increases in tumours, dislocates the tumour cells into the veins and lymphatic vessels associated with the tumours. Interestingly, most of the N-CAM-deficient metastases were located in the lymph nodes, which indicates that the route used by leukocytes is also the route of least resistance for invasion of tumour cells into the vasculature.

Another factor that seems to facilitate tumour metastasis is the growth of tumourassociated lymphatic vessels, which is driven by lymphangiogenic factors of the vascular endothelial growth-factor family, VEGF-C and VEGF-D ${ }^{16-19}$. Notably, transgenic expression of VEGF-C during $\beta$-cell carcinogenesis in RIP1Tag2 mice resulted in the formation of lymph node metastasis ${ }^{16}$. Several interesting questions raised by the present study remain to be explored: Is VEGF-C or VEGF-D upregulated in tumours with reduced amount of N-CAM and which metastasize to the draining regional lymph nodes? What is the role of FGFR-4 upregulation in the carcinomas? Do any of the FGF ligands have a role in the tumour microenvironment?

The work of Cavallaro and colleagues has opened the possibility of a hitherto unknown role for FGFR-4 in tumorigenesis. Expression of a dominant-negative form of FGFR-4 was shown to cause a loss of matrix adhesion comparable to that caused by N-CAM deletion. Perhaps the time has come for mutations in FGFR-4 to be re-examined in human cancers. Kari Alitalo is in the Molecular/Cancer Biology Laboratory, Biomedicium Helsinki, 